\title{
APLICAÇÃO DE BIORREGULADOR NA PRODUTIVIDADE DO ALGODOEIRO E QUALIDADE DE FIBRA ${ }^{1}$
}

\section{APPLICATION OF BIOREGULATOR AND PRODUCTION OF THE COTTON PLANT AND QUALITY OF FIBER}

\author{
Leandro Paiola ALBRECHT ${ }^{2}$ \\ Alessandro de Lucca e BRACCINI ${ }^{3}$ \\ Marizangela Rizzatti ÁVILA ${ }^{4}$ \\ Mauro Cezar BARBOSA ${ }^{5}$ \\ Thiago Toshio RICCI ${ }^{6}$ \\ Alfredo Jr. Paiola ALBRECHT ${ }^{7}$
}

\section{RESUMO}

O objetivo do trabalho foi avaliar a produtividade, componentes de produção da cultura do algodão e a qualidade de fibra do algodoeiro, cultivar de algodão CD 401, em resposta ao uso de biorregulador, via tratamento de sementes e aplicação foliar. Os tratamentos foram compostos por duas formas de aplicação, via tratamento de sementes e via pulverização foliar, em diferentes estádios fenológicos da cultura $\left(V_{3}\right.$ e $\left.B_{1}\right)$, com biorregulador de ação promotora $\left(0,9 \mathrm{~g} \mathrm{dm}^{-3}\right.$

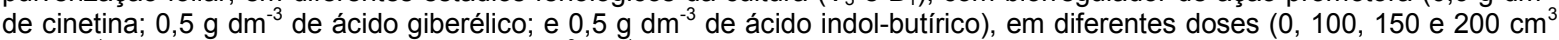
$100 \mathrm{~kg}^{-1}$ de sementes e $0,25,37,5$ e $50 \mathrm{~cm}^{3} \mathrm{ha}^{-1}$, foliar). Foram avaliados, no ano agrícola de 2005/06, a fitotoxicidade, a produtividade de algodão, rendimento de fibra, massa média do capulho e qualidade de fibra. Todas as formas de aplicação do biorregulador aumentaram significativamente a produtividade, o rendimento de fibra, a massa média do capulho e a uniformidade das fibras e não foram fitotóxicas as plantas.

Palavras-chave: Gossypium hirsutum L.; bioestimulante; pluma; rendimento.

\section{ABSTRACT}

The objective of the work was to evaluate the yield components of the cotton culture and the quality of fiber of the cotton plant, in response to the use of a bioregulator, through treatment of seeds and application to foliate. The treatments were composed by two application forms, treatment of seeds and pulverization to foliate, in different stadiums phenologicals of the culture $\left(V_{3}\right.$ e $\left.B_{1}\right)$, with the bioregulator of promotive action $\left(0.9 \mathrm{~g} \mathrm{dm}^{-3}\right.$ cinetin; $0.5 \mathrm{~g} \mathrm{dm}^{-3}$ giberellic acid; and $0.5 \mathrm{~g} \mathrm{dm}^{-3}$ indolbutiric acid), in different doses $\left(0,100,150\right.$ and $200 \mathrm{~cm}^{3} 100 \mathrm{~kg}^{-1}$ of seeds and $0,25,37.5$ and $50 \mathrm{~cm}^{3}$ ha' ${ }^{-1}$, to foliate). The cotton productivity, fiber yield, mass of the cotton boll and fiber quality. Were evaluated all the forms of application of bioregulator increased the productivity significantly, the fiber yield, mass of the cotton boll and uniformity of the fibers and were not phytotoxic to the plants.

Key-words: Gossypium hirsutum L.; plant growth regulator; cotton bolls; performance.

\footnotetext{
${ }^{1}$ Alguns resultados do trabalho foram apresentados no VI Congresso Brasileiro do Algodão, de 13 a 16 de agosto de 2007, Uberlândia - MG. ${ }^{2}$ Engenheiro Agrônomo, Mestre em Agronomia, Doutorando, Bolsista CNPq, do Programa de Pós-Graduação em Agronomia, da Universidade Estadual de Maringá, Maringá, PR - Brasil. E-mail: Ipalbrecht@yahoo.com.br.

${ }^{3}$ Engenheiro Agrônomo, Doutor em Fitotecnia, Professor Associado da Universidade Estadual de Maringá. Av. Colombo, 5790, CEP 87020900, Maringá, PR - Brasil.E-mail: albraccini@uol.com.br. Autor para correspondência.

${ }^{4}$ Engenheira Agrônoma, Doutora em Agronomia, Professora Adjunta da Universidade Estadual de Maringá, Maringá, PR - Brasil. E-mail: marizangela_rizzatti@hotmail.com.

5 Engenheiro Agrônomo, Acadêmico do Mestrado em Agronomia da Universidade Estadual de Maringá, Maringá PR - Brasil. E-mail: mcezarbarbosa@yahoo.com.br.

${ }^{6}$ Acadêmico do curso de Agronomia, Universidade Estadual de Maringá, Maringá, PR - Brasil. E-mail: thiagotoshioricci@hotmail.com.

${ }^{7}$ Acadêmico do curso de Agronomia, Universidade Estadual de Maringá, Maringá, PR - Brasil. E-mail: ajpalbrecht@yahoo.com.br.
} 


\section{INTRODUÇÃO}

$\mathrm{Na}$ busca de melhorias nos atuais níveis de produtividade e redução nos custos de produção do algodoeiro no Brasil, novas tecnologias vêm sendo incorporadas ao sistema de produção. Entre as novas tecnologias em estudo, a manipulação de biorreguladores é uma das estratégias agronômicas para o incremento da produtividade, usadas no controle do crescimento (Hodges et al., 1991; McConnell et al., 1992) ou como bioestimulantes (Santos \& Vieira, 2005).

Reguladores vegetais, usualmente são definidos como compostos orgânicos, não nutrientes, que afetam os processos fisiológicos do crescimento e do desenvolvimento quando aplicados em baixas concentrações. De acordo com Laca-Buendia (1989), reguladores vegetais são substâncias sintetizadas, que aplicadas exogenamente possuem ação análoga aos grupos de hormônios vegetais conhecidos (auxinas, giberelinas, citocininas, retardadores, inibidores e etileno). Vieira \& Castro (2001) adicionam a terminologia do bioestimulante, como sendo uma mistura de dois ou mais reguladores vegetais ou de biorreguladores com outros produtos. Estes podem ser aplicados diretamente nas plantas para alterar os processos vitais e estruturais, com a finalidade de incremento na produção, melhorar a qualidade da fibra e facilitar a colheita (Chiavegato et al., 2007).

Existem no mercado exemplos de produtos comerciais a base de biorregulador, também denominado de bioestimulante, que possuem ação promotora (pois contém em sua composição análogos sintéticos de: auxina, giberelina e citocinina), com a capacidade de estimular o desenvolvimento radicular, aumentando a absorção de água e nutrientes pelas raízes, podendo favorecer também o equilíbrio hormonal da planta (Stoller do Brasil, 1998). Belmont et al. (2003), avaliando o efeito de um bioestimulante comercial sobre a germinação de sementes de três cultivares de algodão, registraram resposta positiva na germinação de sementes. Santos \& Vieira (2005) comprovaram que determinado bioestimulante, aplicado via sementes, é capaz de originar plântulas de algodoeiro mais vigorosas, com maior comprimento, massa seca e porcentagem de emergência em areia e terra vegetal; a área foliar, a altura e o crescimento inicial de plantas de algodão foram incrementados pela aplicação desse biorregulador nas sementes. Enquanto outros autores, como Lima et al. (2006), avaliaram a interação adubação e efeito de biorreguladores com ação promotora na cultura do algodão.

Diante do exposto, a utilização de produtos a base de reguladores vegetais podem não só influenciar na germinação e no desenvolvimento das plântulas de algodão, bem como apresentar efeitos positivos no rendimento da cultura, e incrementar a qualidade da pluma. Contudo, os resultados alcançados até o momento com a utilização de tal tecnologia ainda são relativamente recentes, e não totalmente conclusivos, justificando mais estudos nesse sentido.

Assim, objetivou-se avaliar o uso de biorregulador, via tratamento de sementes e aplicação foliar, sobre a produtividade, alguns componentes de produção e a qualidade de fibra, da cultivar de algodão CD 401, além de observar a fitotoxicidade do produto utilizado.

\section{MATERIAL E MÉTODOS}

O experimento de campo foi instalado na Fazenda Experimental de Iguatemi (FEI), pertencente ao Centro de Ciências Agrárias da Universidade Estadual de Maringá (UEM) e localizada na região noroeste do Estado do Paraná, situada a $52^{\circ} 03^{\prime} 54^{\prime \prime}$ de longitude oeste de Greenwich e $23^{\circ} 22^{\prime} 12^{\prime \prime}$ de latitude sul, com altitude média de $540 \mathrm{~m}$. O solo da área experimental foi classificado como Latossolo Vermelho Eutrófico de textura média (EMBRAPA, 1999). O clima predominante na região é do tipo $\mathrm{Cfa}$, mesotérmico úmido, com chuvas abundantes no verão e inverno seco com verões quentes, segundo classificação de Köppen (IAPAR, 1987). Os dados climáticos de temperatura máxima, mínima e média, assim como umidade relativa e precipitação pluviométrica, foram coletados diariamente, além de outros dados necessários para elaboração do balanço hídrico (Figura 1).

Avaliações de fitotoxicidade foram realizadas a campo, após emergência e posteriores as aplicações foliares, segundo a escala E.W.R.C. (1964). A produtividade de algodão em caroço foi quantificada no Laboratório de Tecnologia de Sementes do Núcleo de Pesquisa Aplicada à Agricultura (NUPAGRI) da UEM, em Maringá - PR, $\mathrm{e}$ as determinações de rendimento e qualidade de fibra realizadas na Cooperativa Central de Pesquisa Agrícola (COODETEC), em Cascavel - PR.

$\mathrm{Na}$ adubação de semeadura foi utilizado 90 $\mathrm{kg} \mathrm{ha}^{-1}$ de $\mathrm{P}_{2} \mathrm{O}_{5}$ na forma de superfosfato simples, $40 \mathrm{~kg} \mathrm{ha}^{-1}$ de nitrogênio $(\mathrm{N})$ na forma de uréia e 40 $\mathrm{kg} \mathrm{ha}^{-1}$ de $\mathrm{K}_{2} \mathrm{O}$ na forma de cloreto de potássio. Foi realizada uma adubação de cobertura com $20 \mathrm{~kg}$ $\mathrm{ha}^{-1}$ de $\mathrm{N}$ na forma uréia, aplicados aos trinta dias após a emergência da cultura. A adubação levou em consideração o laudo de análise de solo e as recomendações técnicas (EMBRAPA, 2001).

O sistema de preparo do solo empregado foi o de plantio direto, na palhada da aveia, semeada no período de inverno e dessecada com glyphosate, trinta dias antes da semeadura do algodão.

As sementes de algodão da cultivar CD 410 (ciclo precoce no Paraná) foram semeadas manualmente em sulcos espaçados de $0,90 \mathrm{~m}$, na profundidade aproximada de $5 \mathrm{~cm}$. As parcelas foram constituídas por oito linhas de $7 \mathrm{~m}$ de comprimento. Para as avaliações, foram consideradas apenas as quatro fileiras centrais, descartando-se $1,0 \mathrm{~m}$ de cada extremidade, totalizando, assim, uma área útil de $18,0 \mathrm{~m}^{2}$.

A semeadura foi realizada no dia 13/10/2005 e os tratamentos foram compostos por duas formas 


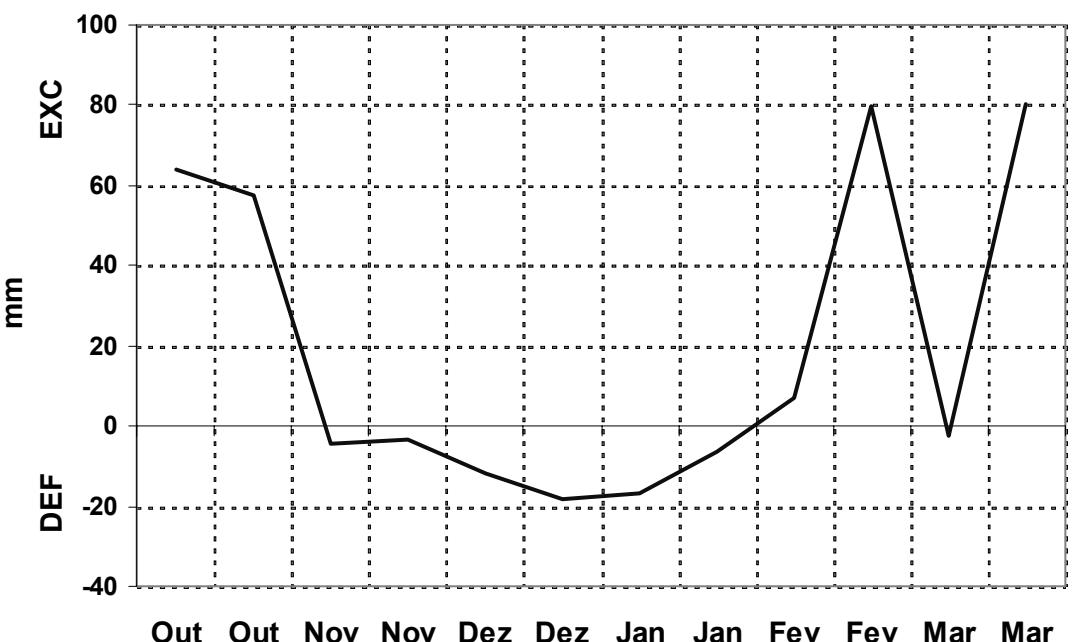

FIGURA 1 - Balanço hídrico da safra 2005/2006, para as condições edafoclimáticas do ensaio. DEF= deficiência; $\mathrm{EXC}=$ excedente.

de aplicação: via tratamento de sementes e pulverização foliar, em diferentes estádios fenológicos da cultura $\left(V_{3}\right.$ e $\left.B_{1}\right)$, com 0 biorregulador Stimulate ${ }^{\circledR}$ dez vezes mais concentrado (10X), em diferentes doses $(0,100$, 150 e $200 \mathrm{~cm}^{3} 100 \mathrm{~kg}^{-1}$ de sementes e 0, 25, 37,5 e $50 \mathrm{~cm}^{3} \mathrm{ha}^{-1}$, foliar). O bioestimulante utilizado é um biorregulador líquido da Stoller do Brasil Ltda., composto, na sua forma concentrada, de $0,9 \mathrm{~g} \mathrm{dm}^{-3}$ de cinetina, $0,5 \mathrm{~g} \mathrm{dm}^{-3}$ de ácido giberélico e $0,5 \mathrm{~g}$ $\mathrm{dm}^{-3}$ de ácido indol-butírico (Stoller do Brasil, 1998).

O tratamento de sementes com o biorregulador foi realizado por ocasião da semeadura, juntamente com aplicação do fungicida Carboxin + Thiram na dosagem de $250 \mathrm{~cm}^{3} 100 \mathrm{~kg}^{-1}$ de sementes $+50 \mathrm{~cm}^{3}$ de água, promovendo-se, por meio da agitação manual, um maior contato entre as sementes e os produtos.

As pulverizações foliares foram efetuadas em dois estádios de desenvolvimento da cultura, ou seja, $\mathrm{V}_{3}$ (terceiro nó vegetativo) e $\mathrm{B}_{1}$ (primeiro botão floral) (Marur \& Ruano, 2001). Na aplicação foliar foi utilizado pulverizador costal propelido a $\mathrm{CO}_{2}$, com pressão constante de $280 \mathrm{kPa}$, equipado com lança contendo 1 bico leque da série Teejet tipo XR 110:02, que, trabalhando a uma altura de $40 \mathrm{~cm}$ do alvo e a uma velocidade de $1 \mathrm{~m} \mathrm{~s}^{-1}$, atingiu uma faixa aplicada de $65 \mathrm{~cm}$ de largura e propiciou um volume de calda de $200 \mathrm{dm}^{3} \mathrm{ha}^{-1}$.

Os tratos culturais foram os mesmos preconizados pelo sistema de produção da região (EMBRAPA, 2001). As plantas daninhas foram controladas por meio de capinas manuais. O controle das principais pragas foi realizado sempre que necessário, com pulverizações sistemáticas de inseticidas como: endossulfam e metamidofós, além de acaricidas, até o final do ciclo da cultura. A área de cultivo encontrava-se isenta de nematóides.
Por ocasião da maturação plena foram efetuadas as seguintes determinações: produtividade de algodão em caroço, rendimento de fibra, massa média do capulho e as características de qualidade de fibra. A colheita foi realizada manualmente, por meio da coleta de todos os capulhos presentes na área útil de cada unidade experimental, em 05 de março de 2006. Partindo-se do rendimento de algodão em caroço nas parcelas, foram estimadas as produtividades em kg ha-1. Para o cálculo da produtividade, o grau de umidade das sementes, determinado por meio do método de estufa a $105 \pm 3{ }^{\circ} \mathrm{C}$ (Brasil, 1992), foi corrigido para $130 \mathrm{~g} \mathrm{ha}^{-1}$ em base úmida.

Foram determinados, o rendimento de fibra e a massa média do capulho. Para avaliação dessas duas características, foram coletados 15 capulhos do terço médio das plantas da área útil de cada parcela. Foi avaliada a massa de 15 capulhos, obtendo-se a massa total e, em seguida, descaroçados, obtendo-se a massa de fibra e a massa de caroço. A massa média do capulho foi obtida subtraindo-se a massa total pelo número de capulhos coletados, sendo os resultados expressos em g. O rendimento de fibra foi obtido pela seguinte equação: $\operatorname{RF}(\%)=M F / M T$ * 100 ; em que:

$\mathrm{RF}=$ rendimento de fibra $(\%) ; \mathrm{MF}=$ massa de fibra (g); MT = massa total dos capulhos (g).

A qualidade de fibra foi avaliada por meio das seguintes características: comprimento $(\mathrm{mm})$, uniformidade (ou \% do índice de uniformidade), resistência (gf tex ${ }^{-1}$ ), elongação (ou alongamento) e índice micronaire (relação entre finura e maturidade da fibra). A classificação do algodão em pluma foi através do High Volume Instrument - HVI (instrumento de alto volume) (Sestren \& Lima, 2007). 
O delineamento experimental utilizado foi em blocos casualizados com quatro repetições. Os dados foram submetidos à análise de variância e as médias, de todos os tratamentos, comparadas pelo método de agrupamento de Scott \& Knott (1974), a $5 \%$ de probabilidade. Enquanto as doses foram analisadas por regressão polinomial a $5 \%$ de probabilidade pelo teste $t$, dentro de cada forma de aplicação (tratamento de sementes e pulverização foliar em $V_{3}$ e $B_{1}$ ) (Banzatto \& Kronka, 2008). Na escolha do melhor modelo de regressão foram adotados os seguintes critérios: regressão significativa, desvios da regressão não-significativa e coeficiente de determinação. Não foi necessária a transformação dos dados, pois todas as pressuposições básicas para análise de variância foram atendidas.

\section{RESULTADOS E DISCUSSÃO}

Analisando a fitotoxicidade, não ocorreram diferenças significativas $(P<0,05)$, pois a nota para todos os tratamentos, após emergência $e$, aplicações foliares, foi 1 (nenhum dano) (E.W.R.C., 1964). Desta forma, o biorregulador, não foi fitotóxico à cultura do algodoeiro, quando aplicado por meio do tratamento de sementes ou por pulverização foliar nos estádios $V_{3}$ e $B_{1}$, independente da dose utilizada.

Houve diferenças significativas $(P<0,05)$ para as variáveis, produtividade de algodão em caroço $\left(\mathrm{kg} \mathrm{ha}^{-1}\right)$, rendimento de fibra e a massa média do capulho, quando foram comparados todos os tratamentos analisados (Tabela 1).

TABELA 1 - Produtividade de algodão em caroço, rendimento de fibra e massa média do capulho da cultivar CD 410, em resposta a diferentes doses e formas de aplicação do biorregulador.

\begin{tabular}{|c|c|c|c|}
\hline \multirow[b]{2}{*}{ Tratamento 1 Dose } & \multicolumn{3}{|c|}{ Características avaliadas $^{2}$} \\
\hline & Produtividade & Rendimento de fibra & Massa média do capulho \\
\hline & --- kg ha ${ }^{-1}$--- & --- \% --- & --- g --- \\
\hline 1. Testemunha $\left(0 \mathrm{~cm}^{3}\right)$ & $1359,84 d$ & $44,86 \mathrm{~b}$ & $4,13 b$ \\
\hline 2. TS $\left(100 \mathrm{~cm}^{3} 100 \mathrm{~kg}^{-1}\right)$ & $1836,12 \mathrm{c}$ & $46,88 a$ & $5,28 \mathrm{a}$ \\
\hline 3. TS $\left(150 \mathrm{~cm}^{3} 100 \mathrm{~kg}^{-1}\right)$ & $2200,60 \mathrm{~b}$ & $47,05 \mathrm{a}$ & 5,97 a \\
\hline 4. TS $\left(200 \mathrm{~cm}^{3} 100 \mathrm{~kg}^{-1}\right)$ & $2341,09 \mathrm{~b}$ & $47,00 \mathrm{a}$ & $5,80 \mathrm{a}$ \\
\hline 5. FL $\left(25 \mathrm{~cm}^{3} \mathrm{ha}^{-1}-\mathrm{V}_{3}\right)$ & $2842,41 \mathrm{a}$ & $46,90 \mathrm{a}$ & $6,03 \mathrm{a}$ \\
\hline 6. FL $\left(37,5 \mathrm{~cm}^{3} \mathrm{ha}^{-1}-\mathrm{V}_{3}\right)$ & 2967,83 a & $47,43 a$ & 5,27 a \\
\hline 7. FL $\left(50 \mathrm{~cm}^{3} \mathrm{ha}^{-1}-\mathrm{V}_{3}\right)$ & $2279,63 \mathrm{~b}$ & $47,51 \mathrm{a}$ & $6,13 \mathrm{a}$ \\
\hline 8. FL $\left(25 \mathrm{~cm}^{3} \mathrm{ha}^{-1}-\mathrm{B}_{1}\right)$ & $2667,37 \mathrm{a}$ & 46,77 a & $5,88 \mathrm{a}$ \\
\hline 9. $\mathrm{FL}\left(37,5 \mathrm{~cm}^{3} \mathrm{ha}^{-1}-\mathrm{B}_{1}\right)$ & $2705,84 \mathrm{a}$ & 47,76 a & $5,64 \mathrm{a}$ \\
\hline 10. FL $\left(50 \mathrm{~cm}^{3} \mathrm{ha}^{-1}-\mathrm{B}_{1}\right)$ & $2283,82 \mathrm{~b}$ & 46,76 a & $5,53 a$ \\
\hline Média & 2348,46 & 46,89 & 5,57 \\
\hline C.V.(\%) & 13,08 & 1,39 & 11,80 \\
\hline
\end{tabular}

$\mathrm{Na}$ Tabela 1 estão apresentados resultados referentes a produtividade de algodão em caroço. A variável produtividade foi a característica avaliada que teve maior influência das doses e formas de aplicação do regulador vegetal, permitindo discriminar os melhores tratamentos, quanto a forma de aplicação (Tabela 1). Observa-se que a maior produtividade média foi obtida pelos tratamentos 5 e $6\left(2.842\right.$ e 2.968 kg ha ${ }^{-1}$, respectivamente) e pelos tratamentos 8 e 9 (2.667 e $2.706 \mathrm{~kg} \mathrm{ha}^{-1}$, respectivamente), ou seja, nas doses de 25 e $35,5 \mathrm{~cm}^{3} \mathrm{ha}^{-1}$ do produto aplicado via foliar nos estádios $V_{3}$ e $B_{1}$, respectivamente. Para a testemunha a produtividade de algodão em caroço foi a menor (de apenas $1.360 \mathrm{~kg} \mathrm{ha}^{-1}$ ).
Os resultados corroboram parcialmente com os dados de Chiavegato et al. (2007), que verificou numericamente que as produções sistematicamente cresceram com o aumento das doses do produto em todas as fases de aplicação. Porém, pelo teste de médias, Chiavegato et al. (2007) constataram que os tratamentos via semente não diferiram estatisticamente do tratamento controle. Esses mesmos autores observaram que, quando 0 produto foi aplicado em $\mathrm{B}_{1}$, o tratamento na maior dose, $50 \mathrm{~cm}^{3} \mathrm{ha}^{-1}$, proporcionou a maior produção de algodão em caroço (cerca de $3.930 \mathrm{~kg} \mathrm{ha}^{-1}$ ), representando um aumento de cerca de $13 \%$ em relação ao tratamento testemunha, ou seja, um incremento real de cerca de $450 \mathrm{~kg} \mathrm{ha}^{-1}$. 
A baixa produtividade média, obtida pela testemunha, neste trabalho, ocorreu em virtude das condições climáticas desfavoráveis observadas no decorrer do desenvolvimento da cultura, que se caracterizaram por período de estiagem, principalmente evidente no mês de dezembro de 2005 e início de janeiro de 2006, observado pelo déficit hídrico (Figura 1). Essas mesmas condições de estresse hídrico, que desencadearam um baixo rendimento da testemunha, por um "strain" plástico (como queda das estruturas reprodutivas) (Taiz \& Zeiger, 2004), propiciaram a expressão mais proeminente dos efeitos benéficos da aplicação do biorregulador, tendo o mesmo, efeito atenuador sobre os efeitos do estresse ambiental.

Vários trabalhos têm mostrado resultados na melhoria da resistência das plantas ao estresse hídrico, quando são submetidas à aplicação de produtos a base de biorreguladores. Os níveis das atividades das enzimas antioxidantes superóxido dismutase (SOD), ascorbato peroxidase (APX) e catalase (CAT) têm sido determinados $e$, de maneira geral, aumentam com o uso de bioestimulantes (Zhang \& Ervin, 2004; Karnok, 2000; Zhang \& Schmidt, 1999). A relação íntima entre a atividade antioxidante e a tolerância ao estresse tem sido identificada em culturas, como milho (Zea mays L.) (Malan et al., 1990) e tabaco (Nicotiana tabacum) (Perl et al., 1993). Outro parâmetro que tem sido melhorado na planta com a aplicação de bioestimulantes é a eficiência fotoquímica (Richardson et al., 2004). A tolerância ao estresse e o incremento fotossintético podem, portanto, gerar elevações na produtividade, como a notada nos resultados observados (Tabela 1).

Castro \& Vieira (2001) usando produto composto por reguladores vegetais de ação promotora verificaram na cultura da soja, plantas com sistemas radiculares mais desenvolvidos, apresentando raízes mais vigorosas com valores de massa seca, crescimento e comprimento total superiores aos encontrados nas plantas não tratadas. Essa condição possibilita uma melhor e maior exploração dos recursos disponíveis no solo, como água e nutrientes minerais, aspecto que certamente influi positivamente na produtividade da cultura. Provavelmente as informações anteriormente citadas (Vieira \& Castro, 2001), são válidas para inúmeras espécies cultivadas, como o feijão (Alleoni et al., 2000) e o próprio algodão (Santos \& Vieira, 2005; Chiavegato et al., 2007). Os resultados em algodão (Tabela 1) são valiosos na comprovação da eficácia agronômica de biorreguladores, tidos como bioestimulantes, na cultura do algodão.

De acordo com Ávila et al. (2008), trabalhando com a cultivar de soja CD 202, a maior produtividade média $\left(3.175 \mathrm{~kg} \mathrm{ha}^{-1}\right)$ foi obtida pelo tratamento com a maior dose do mesmo biorregulador utilizado nesse experimento aplicado via foliar $\left(75 \mathrm{~cm}^{3} \mathrm{ha}^{-1}\right)$, promovendo um incremento no rendimento da cultura superior a $92 \%$, em relação à testemunha não tratada, na qual a produtividade foi de apenas $1.649 \mathrm{~kg} \mathrm{ha}^{-1}$. Contribuindo na afirmativa de que há uma eficácia fisiológica no uso do regulador vegetal em questão, o que potencializa a produção vegetal, não só para a soja, mas também para o algodão (Tabela 1).

$\mathrm{Na}$ Tabela 2, observa-se a regressão polinomial, para a variável produtividade de algodão em caroço, sob efeito de aplicações foliares $\left(V_{3} e\right.$ $B_{1}$ ). A tendência para ambas as aplicações foi quadrática, em que na aplicação em $V_{3}$, o ponto de máximo foi de $30 \mathrm{~cm}^{3} \mathrm{ha}^{-1}$ e o máximo da função foi de $2961,72 \mathrm{~kg} \mathrm{ha-1}$ de algodão em caroço. Enquanto para o estádio $\mathrm{B}_{1}$, a dose máxima foi de $31,82 \mathrm{~cm}^{3} \mathrm{ha}^{-1} \mathrm{e}$ a produtividade máxima de $2739,01 \mathrm{~kg} \mathrm{ha}{ }^{-1}$. Portanto, aplicações foliares, especialmente em $V_{3}$, são mais satisfatórias, gerando os maiores rendimento em doses próximas de $30 \mathrm{~cm}^{3} \mathrm{ha}^{-1}$.

TABELA 2 - Equações de regressão polinomial, ajustadas para as diferentes variáveis avaliadas, da cultivar CD 410, em resposta a diferentes doses e formas de aplicação do biorregulador.

\begin{tabular}{llll}
\hline Variável & Forma de aplicação $^{1}$ & $\mathrm{R}^{2}$ & Equação \\
\hline Produtividade & Foliar em $\mathrm{V}_{3}$ & 0,9917 & $\hat{\mathrm{Y}}=1348,8+105,49^{*} \mathrm{X}-1,7242^{\star} \mathrm{X}^{2}$ \\
Produtividade & Foliar em $\mathrm{B}_{1}$ & 0,9917 & $\hat{\mathrm{Y}}=1358,5+86,781^{\star} \mathrm{X}-1,3638^{\star} \mathrm{X}^{2}$ \\
Produtividade & Tratamento de sementes & 0,9854 & $\hat{\mathrm{Y}}=1361,3+5,0938^{\star} \mathrm{X}$ \\
Rendimento de Fibra & Foliar em $\mathrm{V}_{3}$ & 0,9085 & $\hat{\mathrm{Y}}=45,118+0,0138^{*} \mathrm{X}$ \\
Uniformidade & Foliar em $\mathrm{V}_{3}$ & 0,9443 & $\hat{\mathrm{Y}}=81,485+0,0501^{\star} \mathrm{X}$ \\
Uniformidade & Tratamento de sementes & 0,8981 & $\hat{\mathrm{Y}}=81,561+0,0521^{\star} \mathrm{X}$ \\
Comprimento & Tratamento de sementes & 0,9548 & $\hat{\mathrm{Y}}=27,959+0,0108^{\star} \mathrm{X}$ \\
\hline
\end{tabular}

${ }^{1}$ Somente foram ajustados modelos de regressão polinomial para as formas de aplicação, dentro de cada variável, quando significativos a $5 \%$ de probabilidade pelo teste $t$. 
ALBRECHT, L.P. et al. Aplicação de biorregulador na produtividade...

No entanto, altas doses, via foliar, do biorregulador, foram negativas a fisiologia da planta, pois revertem em decréscimos no rendimento de algodão em caroço, observada pela tendência quadrática na produtividade para aplicações feitas via foliar (Tabela 2). Tal efeito denota que, doses crescentes tem um limite no tocante ao efeito promotor, ultrapassando determinado ponto, ocorrem efeitos de antagonismo ao crescimento e desenvolvimento vegetal, provavelmente em função do desbalanço hormonal, conforme sugere a literatura (Vieira \& Castro, 2001; Taiz \& Zeiger, 2004; Moterle et al., 2008).

$\mathrm{Na}$ Tabela 2, observa-se efeito significativo, com tendência linear crescente, para variável produtividade, sob efeito do tratamento de sementes. Ocorreu um incremento de $5,0938 \mathrm{~kg}$ $\mathrm{ha}^{-1}$ (coeficiente angular), para cada $\mathrm{cm}^{3} \mathrm{ha}^{-1} \mathrm{do}$ regulador vegetal aplicado; até um máximo de $2380,06 \mathrm{~kg} \mathrm{ha}^{-1}$ (na dose de $200 \mathrm{~cm}^{3} \mathrm{ha}^{-1}$ ). Resultados que podem viabilizar o posicionamento de tratamento de sementes com biorregulador, até um limiar econômico, como uma opção na elevação de produtividades de algodão em caroço.

Os resultados obtidos no rendimento de fibra e massa média do capulho (Tabela 1 ) revelaram efeito significativo de todas as formas de aplicação avaliadas, com o regulador vegetal, em que somente a testemunha, sem biorregulador, foi inferior aos demais. Portanto, ocorreram ganhos no peso médio do capulho e rendimentos de fibra, promovidos pelos tratamentos com o produto de controle hormonal, independente da forma de aplicação.

No que concerne ao rendimento de fibra e massa média de capulho, somente a variável rendimento de fibra, na aplicação foliar em $V_{3}$ de biorregulador, apresentou comportamento linear crescente em função das doses, pela análise de regressão (Tabela 2). Com uma elevação no rendimento de $0,0138 \%$ a cada $\mathrm{cm}^{3}$ ha ${ }^{-1}$ do produto aplicado, com rendimento, na dose de $50 \mathrm{~cm}^{3} \mathrm{ha}^{-1}$, de $45,808 \%$ de fibra. Tais resultados, fornecidos pelo experimento, solidificam recomendações de aplicação do biorregulador, via foliar, em $V_{3}$, pois além do acréscimo na produtividade, há aumento no rendimento de fibra, fornecendo maior renda aos cotonicultores.

$\mathrm{Na}$ Tabela 3 estão apresentados os resultados obtidos nas diferentes características de qualidade de fibra, em resposta aos tratamentos com doses e formas de aplicação do biorregulador na cultura do algodoeiro. Observa-se que apenas as características de comprimento e uniformidade de fibra apresentaram resultados significativos pela análise de variância dos dados.

TABELA 3 - Características de qualidade de fibra de algodão da cultivar CD 410, em resposta a diferentes doses e formas de aplicação do biorregulador.

\begin{tabular}{|c|c|c|c|c|c|}
\hline \multirow[b]{2}{*}{ Tratamento $1 /$ Dose } & \multicolumn{5}{|c|}{ Características avaliadas $^{2}$} \\
\hline & Comprimento & Uniformidade & Resistência & Elongação & Micronaire \\
\hline & --- mm --- & -- \% (UI) -- & --- gf tex ${ }^{-1}$--- & --- \% --- & -- Índice -- \\
\hline 1. Testemunha $\left(0 \mathrm{~cm}^{3}\right)$ & $27,850 \mathrm{~b}$ & $81,300 \mathrm{~b}$ & $26,250 \mathrm{a}$ & $5,200 \mathrm{a}$ & $4,250 \mathrm{a}$ \\
\hline 2. TS $\left(100 \mathrm{~cm}^{3} 100 \mathrm{~kg}^{-1}\right)$ & 29,325 a & 83,275 a & $29,100 \mathrm{a}$ & $5,575 a$ & $4,850 \mathrm{a}$ \\
\hline 3. TS $\left(150 \mathrm{~cm}^{3} 100 \mathrm{~kg}^{-1}\right)$ & 29,425 a & 83,725 a & $29,700 \mathrm{a}$ & $5,650 \mathrm{a}$ & $4,675 \mathrm{a}$ \\
\hline 4. TS $\left(200 \mathrm{~cm}^{3} 100 \mathrm{~kg}^{-1}\right)$ & 30,075 a & 83,800 a & 30,625 a & $5,725 a$ & $4,725 \mathrm{a}$ \\
\hline 5. FL $\left(25 \mathrm{~cm}^{3} \mathrm{ha}^{-1}-V_{3}\right)$ & 29,425 a & $83,050 \mathrm{a}$ & 29,825 a & $5,600 \mathrm{a}$ & $4,700 \mathrm{a}$ \\
\hline 6. FL $\left(37,5 \mathrm{~cm}^{3} \mathrm{ha}^{-1}-V_{3}\right)$ & $28,950 \mathrm{~b}$ & 83,475 a & 28,375 a & 5,625 a & $4,775 \mathrm{a}$ \\
\hline 7. FL $\left(50 \mathrm{~cm}^{3} \mathrm{ha}^{-1}-V_{3}\right)$ & $29,500 \mathrm{a}$ & $83,750 \mathrm{a}$ & $29,525 \mathrm{a}$ & $5,775 a$ & $4,750 \mathrm{a}$ \\
\hline $\begin{array}{l}\text { 8. FL }\left(25 \mathrm{~cm}^{3} \mathrm{ha}^{-1}-\mathrm{B}_{1}\right) \\
\text { 9. FL }\left(37,5 \mathrm{~cm}^{3} \mathrm{ha}^{-1}-\mathrm{B}_{1}\right)\end{array}$ & $\begin{array}{l}28,700 b \\
28,650 b\end{array}$ & $\begin{array}{l}83,800 \mathrm{a} \\
83,100 \mathrm{a}\end{array}$ & $\begin{array}{l}28,675 \text { a } \\
29,350 \mathrm{a}\end{array}$ & $\begin{array}{l}5,575 \text { a } \\
5,500 \text { a }\end{array}$ & $\begin{array}{l}4,675 a \\
4,550 a\end{array}$ \\
\hline 10. FL $\left(50 \mathrm{~cm}^{3} \mathrm{ha}^{-1}-\mathrm{B}_{1}\right)$ & 28,775 b & $82,850 \mathrm{a}$ & $27,700 \mathrm{a}$ & $5,475 a$ & $4,650 \mathrm{a}$ \\
\hline Média & 29,068 & 83,213 & 28,913 & 5,570 & 4,660 \\
\hline C.V.(\%) & 2,36 & 1,17 & 6,49 & 3,86 & 5,33 \\
\hline
\end{tabular}


O tratamento das sementes com o biorregulador, e a aplicação foliar do produto no estádio $V_{3}$, superou significativamente 0 comprimento das fibras nas demais formas de aplicação. Contudo, apenas nas doses de 25 e 50 $\mathrm{cm}^{3} \mathrm{ha}^{-1}$ da aplicação foliar do regulador de crescimento no estádio $V_{3}$ ocorreu diferença significativa no comprimento das fibras. Os demais tratamentos não diferiram significativamente na referida característica.

No tocante à uniformidade das fibras, todas as formas de aplicação avaliadas no presente trabalho apresentaram diferenças significativas, ou seja, tanto o tratamento de sementes, quanto a pulverização foliar do biorregulador nos estádios de desenvolvimento $V_{3}$ e $B_{1}$, independente da dose aplicada, melhoraram significativamente a uniformidade das fibras.

$\mathrm{Na}$ análise de regressão para a variável uniformidade de fibra (aplicação foliar no $V_{3}$ e tratamento de sementes) e comprimento de fibra (tratamento de sementes); todas as equações ajustadas apresentaram tendência linear crescente (Tabela 2), ou seja, elevações na dose do biorregulador aumentam a qualidade da fibra, quanto a uniformidade (aplicação foliar e tratamento de sementes) e comprimento (tratamento de sementes).

Com relação ao comprimento das fibras e sua uniformidade, ocorre uma forte relação com outras características das fibras e da massa dos fios têxteis, conseqüentemente influenciam nos processos subseqüentes à fiação $e$ as características dos tecidos (Sestren \& Lima, 2007). Desde modo, biorreguladores, tidos como bioestimulantes, podem contribuir na cadeia produtiva, gerando produtos com maior aptidão têxtil e, por conseguinte, aumentando a rentabilidade dos produtores da fibra, já que agrega mais valor ao produto.

Portanto, o uso de biorreguladores com ação promotora, são passíveis de serem recomendados para uso na cotonicultura, focando elevação na qualidade da fibra, na produtividade e nos componentes de produção, conforme resultados do presente trabalho. No entanto, quando a aplicação for via foliar, doses elevadas podem ser prejudiciais, e são mais responsivas quando aplicadas no estádio vegetativo.

\section{CONCLUSÕES}

O uso de biorregulador possibilita incrementos na produtividade de algodão em caroço, no rendimento de fibra, massa média do capulho e em atributos da qualidade de fibra e, não são fitotóxicas a planta.

\section{CONFLITOS DE INTERESSES}

Não há nenhuma relação comercial dos autores com a fabricante do produto comercial citado neste artigo.

\section{REFERÊNCIAS}

1. ALLEONI, B.; BOSQUEIRO, M.; ROSSI, M. Efeito de reguladores vegetais de Stimulate no desenvolvimento e produtividade do feijoeiro. Publicatio UEPG, v. 1, n. 6, p. 23-35, 2000.

2. ÁVILA, M. R. et al. Bioregulador application, agronomic efficiency, and quality of soybean seeds. Scientia Agricola, v. 65 , n. 6, p. 604-612, 2008.

3. BANZATTO, D. A.; KRONKA, S. N. Experimentação Agrícola. 3. ed. Jaboticabal: FUNEP, 2008. 237 p.

4. BELMONT, K. P. de C. et al. Ação de fitorregulador de crescimento na germinação de sementes de algodoeiro. In: CONGRESSO BRASILEIRO DE ALGODÃO, 4., 2003, Goiânia. Anais... Goiânia: ABRAPA, AMIPA, EMBRAPA Algodão, 2003. 4 p. (CD-ROM).

5. BRASIL - Ministério da Agricultura e Reforma Agrária. Regras para análise de sementes. Brasília: SNDA/DNDV/CLAV, 1992. 365 p.

6. CASTRO, P. R. C.; VIEIRA, E. L. Aplicações de reguladores vegetais na agricultura tropical. Guaíba: Livraria e Editora Agropecuária, 2001. $132 \mathrm{p}$.

7. CHIAVEGATO, E. J. et al. Efeito de Stimulate 10X em diferentes doses e estádios de aplicação via foliar e tratamento de sementes em algodoeiro. In: CONGRESSO BRASILEIRO DE ALGODÃO, 6., 2007, Uberlândia. Anais... Uberlândia: ABRAPA, AMIPA, EMBRAPA Algodão, 2007. 4 p. (CD-ROM).

8. EMPRESA BRASILEIRA DE PESQUISA AGROPECUÁRIA (EMBRAPA). Sistema brasileiro de classificação dos solos. Brasília: EMBRAPA, 1999. 412 p.

9. EMPRESA BRASILEIRA DE PESQUISA AGROPECUÁRIA (EMBRAPA). Algodão: tecnologia de produção. Dourados: EMBRAPA Agropecuária Oeste, 2001. 296 p.

10. EUROPEAN WEED REARCH COUNCIL (E.W.R.C.) Report of 3nd and 4nd meeting of E.W.R.C. Cittee of methods in weed research. Weed Research, v. 4, n. 1, p. 88, 1964.

11. HODGES, H. F.; REDDY, V. R.; REDDY, K. R. Mepiquat chloride and temperature effects on photosynthesis and respiration of fruiting cotton. Crop Science, v. 31, n. 5, p. 1302-1308, 1991.

12. INSTITUTO AGRONÔMICO DO PARANÁ (IAPAR). Cartas climáticas básicas do Estado do Paraná. Londrina: Instituto Agronômico do Paraná, 1987. 35 p.

13. KARNOK, K. J. Promises, promises: can biostimulants deliver? Golf Course Management, v. 68, n. 8, p. 67-71, 2000.

14. LACA-BUENDIA, J. P. Efeito de doses de reguladores de crescimento em algodoeiro (Gossypium hirsutum L.). Revista Brasileira de Fisiologia Vegetal, v. 1, n. 1, p. 109-113, 1989.

15. LIMA, M. M. et al. Nitrogênio e promotor de crescimento: efeitos no crescimento e desenvolvimento do algodão colorido verde. Revista Brasileira Engenharia Agrícola e Ambiental, v. 10, n. 3, p. 624-628, 2006.

16. MALAN, C.; GREYLING, M. M; GRESSEL, J. Correlation between Cu/Zn superoxide dismutase and glutathione reductase, and environmental and xenobiotic stress tolerance in maize inbreeds. Plant Science, v. 69, n. 2, p. 157-166, 1990 
ALBRECHT, L.P. et al. Aplicação de biorregulador na produtividade...

17. MARUR, C. J.; RUANO, O. A. A reference system for determination of cotton plant development. Revista de Oleaginosas e Fibrosas, v. 5, n. 2, p. 243-247, 2001

18. McCONNELL, J. S. et al. Response of cotton to nitrogen fertilization and early multiple aplications of mepiquat chloride. Journal of Plant Nutrition, v. 15, n. 4, p. 457-468, 1992

19. MOTERLE, L. M. et al. Efeito da aplicação de biorregulador no desempenho agronômico e produtividade da soja. Acta Scientiarum Agronomy, v. 30, n. 5, p. 701-709, 2008.

20. PERL, A. et al. Enhanced oxidative-stress defense in transgenic potato expressing tomato $\mathrm{Cu}, \mathrm{Zn}$ superoxide dismutases. Theoretical and Applied Genetics, v. 85, n. 5, p. 568-576, 1993.

21. RICHARDSON, A. D. et al. Drought stress and paper birch (Betula papyrifera) seedlings: effects of an organic biostimulant on plant health and stress tolerance, and detection of stress effects with instrument-based, noninvasive methods. Journal of Arboriculture, v. 30, n. 1, p. 52-61, 2004

22. SANTOS, C. M. G.; VIEIRA, E. L. Efeito de bioestimulante na germinação de sementes, vigor de plântulas e crescimento inicial do algodoeiro. Magistra, v. 17, n. 3, p. 124-130, 2005.

23. SCOTT, A.; KNOTT, M. Cluster-analysis method for grouping means in analysis of variance. Biometrics, v. 30, n. 3 , p. 507-512, 1974.

24. SESTREN, J. A.; LIMA, J. J. Características e classificação da fibra de algodão. In: FREIRE, E. C. (Ed.). Algodão no cerrado do Brasil. Brasília: ABRAPA, 2007. p. 765-820.

25. STOLLER DO BRASIL. Stimulate Mo em hortaliças: informativo técnico. Cosmópolis: Stoller do Brasil, Divisão Arbore, 1998. 1 p.

26. TAIZ, L.; ZEIGER, E. Crescimento e desenvolvimento. In: TAIZ, L.; ZEIGER. Fisiologia vegetal. 3. ed.Tradução E. R. Santarém. Porto Alegre: Artmed, 2004. p. 335-642.

27. VIEIRA, E. L.; CASTRO, P. R. C. Ação de bioestimulante na germinação de sementes, vigor de plântulas, crescimento radicular e produtividade de soja. Revista Brasileira de Sementes, v. 23, n. 2, p. 222-228, 2001.

28. ZHANG, X.; ERVIN, E. Cytokinin-containing seaweed and humic acid extracts associated with creeping bentgrass leaf cytokinins and drought resistance. Crop Science, v. 44, n. 5, p. 1737-1745, 2004.

29. ZHANG, X.; SCHMIDT, R. E. Antioxidant response to hormone-containing product in Kentucky bluegrass subjected to drought. Crop Science, v. 39, n. 6, p. 545-551, 1999. 